\title{
Temporal Series Analysis on Avoidable Mortality for the Assessment of an Intervention Program in a Hospital
}

\author{
José L. Alfonso-Sanchez ${ }^{1,2^{*}}$, Belen Alfonso-Landete ${ }^{1}$, Isabel Martinez-Martinez ${ }^{3}$ \\ 'Department of Preventive Medicine and Public Health, School of Medicine, University of Valencia, Valencia, Spain \\ ${ }^{2}$ Preventive Medicine and Occupational Risk Prevention Services, University General Hospital of Valencia, Valencia, Spain \\ ${ }^{3}$ Nursery College, Universidad de Valencia, Valencia, Spain
}

*Corresponding Author: José L. Alfonso-Sanchez, M.D., Professor, Department of Preventive Medicine and Public Health, School of Medicine, University of Valencia, Av. Blasco Ibáñez, 13. 46010 Valencia. Spain. Head of Preventive Medicine Service, University General Hospital of Valencia, Av. de las Tres Cruces, 2. 46014 Valencia, Spain. Tel: +34-961972250, +34650715973; Email: jose.I.alfonso@uv.es

Received January 1, 2017; Accepted January 17, 2017; Online Published February 19, 2017

\begin{abstract}
Background: Avoidable mortality (AM) is one of the most important health indicators (HI) and represents the quality of care in a hospital.

Objective: This study measured the efficacy of a training program for a hospital healthcare staff to reduce AM.

Methods: This epidemiological study on community intervention analyzed time-series data on HI by semesters from 2008 to 2015. The pre-intervention phase was examined from January 2008 to December 2014; the intervention phase was investigated in the first semester of 2015; and the post-intervention phase was examined in the second semester of 2015. Results: Resindicate a series with a rising tendency until the 14th semester and a pronounced descent in the 16th semester. The relative variation rate (RVR) was $-20 \%$ to $+20 \%$ with some exceptions. HI was $0.53 \%$ in the 16 th semester rather than the expected $0.70 \%$ observed in the pre-intervention phase; therefore, $0.17 \%$ additional deaths were avoided because of the training seminar.

Conclusion: The positive results suggest that this strategy is an important element in decreasing avoidable deaths in hospitals. Keywords: Health services, Quality of health care, Cause of death
\end{abstract}

\section{Background}

Health indicators comprise a list of causes or pathologies for avoidable mortality (AM) used to measure the quality of specialized care in hospitals. Researchers such as Rutstein et al in 1976 proposed counting the cases of diseases, disabilities, and unnecessary or premature deaths as a measure of quality in healthcare. ${ }^{1}$ They identified 91 processes and illnesses. In the 1980s, this line of research was developed further by Charlton et $\mathrm{al}^{2}{ }^{2}$ who examined 14 of the pathologies from Rutstein's list which were unanimously accepted as causes of deaths that could be avoided with timely healthcare. This list has varied over time due to fluctuations in incidence, the severity of different pathologies, and advances in the field of medicine. ${ }^{3}$

Avoidable deaths, then, are those deaths caused by specific diseases within selected age groups that could have been completely or substantially avoided if appropriate access to medical care was provided.
The causes of AM are divided into two groups. The first group comprises causes largely susceptible to primary prevention at a national level, denominated as those susceptible to intersectoral health policy interventions. The second group is made up of diseases which require specialized secondary care, better known as health indicators (HI), which include tuberculosis, cancer of the cervix and uterus, Hodgkin disease, chronic rheumatic heart disease, respiratory disease, asthma, appendicitis, abdominal hernia, cholecystectomy, arterial hypertension and cerebrovascular stroke, maternal and perinatal mortality, pertussis, measles, osteomyelitis, and periostitis. ${ }^{4,5}$

AM in Spain has decreased in the past 2 decades with improvement in the hospital conditions and health policies of different regions. Another benefit of these improvements is the lengthening of life expectancy for men and women. ${ }^{6,7}$ Studies that compared mortality due to avoidable and unavoidable causes from 1981 to 2001 showed a reduction

Copyright (C) 2017 The Hospital Practices and Research. This is an open-access article distributed under the terms of the Creative Commons Attribution License (http://creativecommons.org/licenses/by/4.0), which permits unrestricted use, distribution, and reproduction in any medium, provided the original work is properly cited. 
of -1.68 for the former and -1.28 for the latter. ${ }^{6}$

From 1990 to 2004, the Valencia region experienced a larger drop in AM than in general mortality, in line with indicators in the rest of the country and elsewhere in Europe. There was also a notable decrease in deaths due to hypertension, cerebrovascular diseases, and tuberculosis, especially among men. This benefit was attributed in large part to primary prevention measures and advances in treatments. ${ }^{8}$

The analysis of AM as a time series has two objectives: (a) to explain the variations observed to determine if they respond to a behavioral pattern; and $(b)$ to predict the course of future measurements.

\section{Objective}

During this study, a staff training program was implemented for healthcare professionals as a method of intervention with the purpose of decreasing AM. The objective of this study was to measure the efficacy of that intervention.

\section{Methods}

This is an epidemiological study on community intervention which consisted of carrying out a training seminar for healthcare staff in a third level hospital in the Valencian region. Data was collected from the Minimum Basic Data Set (MBDS), from January 1, 2008 to December 31, 2015 and was divided by semesters into the pre-intervention phase (semesters 1-14, from January to December 2014); the intervention phase in which the training program was implemented (semester 15, from January to June 2015); and the post-intervention phase which measured the effects of the intervention program (semester 16, from July to December 2015).

Inclusion criteria: All patients of the corresponding health department who were admitted with a principal diagnosis of CIE-9-CM of $\mathrm{HI}$ and registered in the hospital's MBDS during the study period were included. The variables observed included age, date of admission, principal diagnosis, and circumstances of discharge. The list of AM causes used was from Holland's classification. ${ }^{9}$

Exclusion criteria were patients referred to other hospitals and outpatients.

The training course was carried out with a doubleblind implementation from January through June 2015. It consisted of practical training in follow-up, intervention, and prevention of complications for pathologies on the $\mathrm{AM}$ list, and it was directed at the physician and nursing staff.

The automatic jump detection method was applied to detect jumps. When it tested positive, the results were corrected by the log-linear model of the general form model (see Appendix). The general rate of seasonal variation of the series was calculated. It was divided into even and odd semesters.

Finally, the relative variation rate (RVR) of the series was determined, ${ }^{10-12}$ and the multiple logistic regression and the Hosmer-Lemeshow test were applied. Statistical differences were $P=.05$.

The first objective in the time-series analysis of AM was to explain the variation observed in the number of deaths in the series and determine if they corresponded to a pattern of behavior.

The second objective of the study was to calculate through multiple logistical regression the percentage of deaths which would have been observed in the 16th semester if no training course had been carried out.

\section{Results}

The base population of the hospital was 468000 inhabitants. The hospital has all medical and surgical specialties in addition to a maternity and pediatric building. It is located on the Mediterranean coast.

Table 1 shows total hospital admissions and those admissions eliminated because of not meeting the inclusion criteria. The last groups were classified into 2 categories: HI pathologies and other pathologies. An increase in the percentage of $\mathrm{HI}$ pathologies over total pathologies of admitted patients was observed. In the first semester of 2008 , these diseases accounted for $16 \%$ of the total; this figure rose to $19 \%$ in the last semester studied.

Table 2 shows the total numbers of admissions and deaths of each HI pathology over 16 semesters. The largest number of deaths during the study period was caused by hypertension and cerebrovascular diseases, with a minimum of 3 and a maximum of 11 deaths each semester. This was followed by abdominal hernia with 14 deaths and cancer of the uterus with 6 deaths. The remaining categories showed a range of 1 to 4 deaths during the 16 -semester study period.

Furthermore, a statistically significant difference was observed between the total number of deaths in the 14th semester (the last in the pre-intervention phase) and the total number of deaths in the 16th semester (corresponding to the post-intervention phase $[P<.000])$.

Table 3 shows the calculated values in the total mortality due to HI series and the values of tendency in hypertension and cerebrovascular diseases.

By calculating tendency using the rolling average methodology, the series was "softened," and the principal component of oscillations in the short and medium term and seasonal, cyclical, residual, and accidental variations were excluded. To compare successive values of the series, the seasonal component was eliminated.

The procedure, carried out with rolling averages, defined the seasonality and residual variations, allowing investigators to calculate the general rate of seasonal variation (93.87), which corresponds to the semesters between January and June, 2008 to 2015, as well as the general rate of seasonal variation (107.13) which corresponds to the semesters between July and December of the same period. Rates 1 and 2 are values which approximate 100 due to seasonal effects. The first semesters present a rate near the annual average, while the rates in the second semester are slightly higher. Because these values were not equal to 100 , it can be concluded that the series has seasonality.

Over the course of the study period, the highest number of deaths occurred because of hypertension and cerebrovascular diseases; thus, the rolling average method 


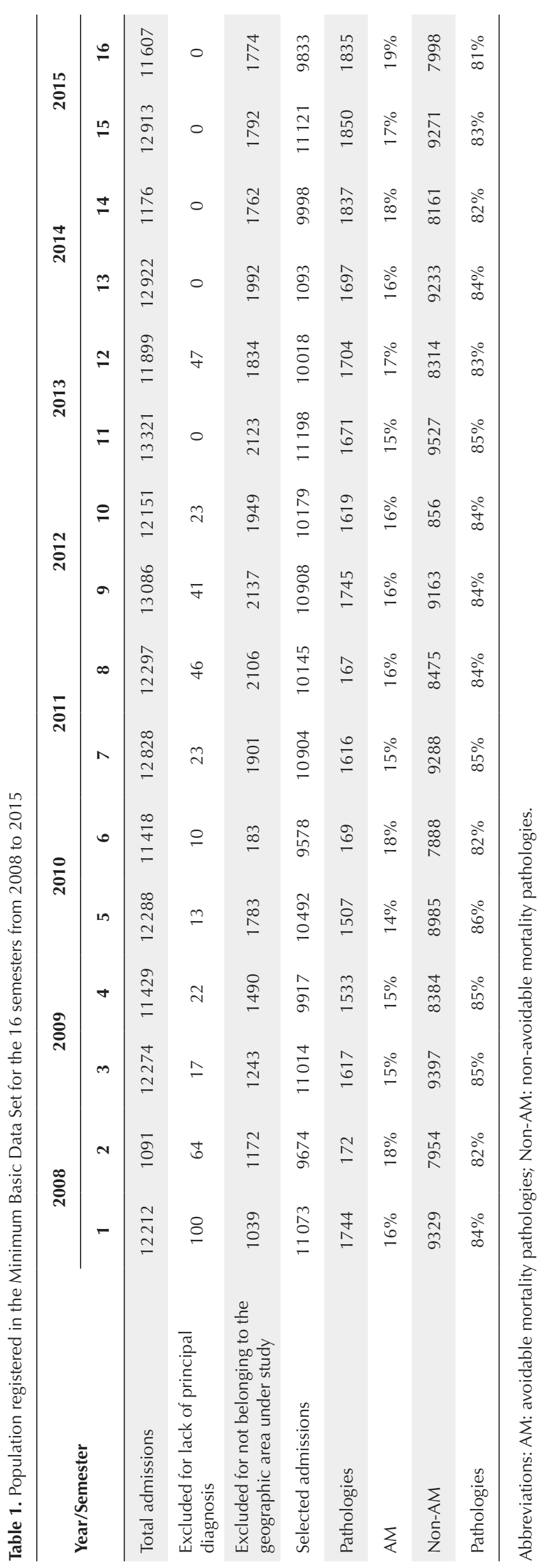

4 | Hospital Practices and Research 2017;2(1):2-8 
Table 2. Number of Admissions and Total Deaths Due to HI Pathologies in the 16 Semesters From 2008 to 2015

\begin{tabular}{|c|c|c|c|c|c|c|c|c|c|c|c|c|c|c|c|c|}
\hline \multirow{2}{*}{ HI Category } & \multicolumn{2}{|c|}{2008} & \multicolumn{2}{|c|}{2009} & \multicolumn{2}{|c|}{2010} & \multicolumn{2}{|c|}{2011} & \multicolumn{2}{|c|}{2012} & \multicolumn{2}{|c|}{2013} & \multicolumn{2}{|c|}{2014} & \multicolumn{2}{|c|}{2015} \\
\hline & 1 & 2 & 3 & 4 & 5 & 6 & 7 & 8 & 9 & 10 & 11 & 12 & 13 & 14 & 15 & 16 \\
\hline \multirow{2}{*}{ Typhoid fever } & 0 & 1 & 0 & 0 & 0 & 1 & 0 & 0 & 0 & 0 & 0 & 0 & 1 & 0 & 1 & 0 \\
\hline & 0 & 0 & 0 & 0 & 0 & 0 & 0 & 0 & 0 & 0 & 0 & 0 & 0 & 0 & 0 & 0 \\
\hline \multirow{2}{*}{$\begin{array}{l}\text { Tuberculosis and late } \\
\text { onset effects }\end{array}$} & 26 & 24 & 16 & 13 & 16 & 12 & 22 & 19 & 14 & 12 & 10 & 15 & 17 & 24 & 23 & 12 \\
\hline & 0 & 1 & 0 & 0 & 0 & 0 & 0 & 1 & 1 & 0 & 0 & 0 & 0 & 1 & 0 & 0 \\
\hline \multirow{2}{*}{ Pertussis } & 2 & 9 & 0 & 0 & 0 & 0 & 0 & 0 & 0 & 0 & 0 & 0 & 0 & 0 & 0 & 0 \\
\hline & 0 & 0 & 0 & 0 & 0 & 0 & 0 & 0 & 0 & 0 & 0 & 0 & 0 & 0 & 0 & 0 \\
\hline \multirow{2}{*}{$\begin{array}{l}\text { Cancer of the cervix } \\
\text { and body }\end{array}$} & 4 & 2 & 2 & 0 & 4 & 3 & 3 & 3 & 3 & 1 & 3 & 4 & 0 & 1 & 1 & 5 \\
\hline & 0 & 0 & 0 & 0 & 0 & 0 & 0 & 0 & 0 & 0 & 0 & 1 & 0 & 0 & 0 & 0 \\
\hline \multirow{2}{*}{ Cancer of the cervix } & 7 & 6 & 3 & 1 & 3 & 3 & 6 & 9 & 5 & 5 & 10 & 0 & 0 & 5 & 2 & 3 \\
\hline & 0 & 0 & 0 & 0 & 1 & 0 & 0 & 1 & 0 & 0 & 1 & 0 & 0 & 1 & 1 & 1 \\
\hline \multirow{2}{*}{ Hodgkin disease } & 4 & 2 & 5 & 0 & 6 & 3 & 1 & 4 & 3 & 3 & 5 & 2 & 3 & 2 & 3 & 3 \\
\hline & 0 & 0 & 0 & 0 & 0 & 0 & 0 & 0 & 0 & 0 & 1 & 0 & 0 & 0 & 0 & 0 \\
\hline \multirow{2}{*}{$\begin{array}{l}\text { Chronic rheumatic } \\
\text { heart disease }\end{array}$} & 4 & 2 & 4 & 9 & 4 & 2 & 2 & 2 & 1 & 2 & 2 & 4 & 3 & 1 & 1 & 0 \\
\hline & 0 & 0 & 0 & 0 & 0 & 0 & 0 & 0 & 0 & 0 & 0 & 1 & 0 & 0 & 0 & 0 \\
\hline \multirow{2}{*}{$\begin{array}{l}\text { Hypertension and } \\
\text { cerebrovascular }\end{array}$} & 130 & 93 & 103 & 84 & 87 & 106 & 110 & 89 & 112 & 90 & 72 & 79 & 82 & 91 & 113 & 80 \\
\hline & 11 & 4 & 5 & 7 & 3 & 6 & 7 & 5 & 8 & 8 & 8 & 9 & 9 & 9 & 5 & 4 \\
\hline \multirow{2}{*}{ Respiratory diseases } & 248 & 226 & 206 & 135 & 203 & 170 & 153 & 142 & 166 & 149 & 182 & 117 & 191 & 117 & 142 & 160 \\
\hline & 0 & 1 & 0 & 0 & 0 & 0 & 0 & 0 & 0 & 0 & 0 & 0 & 0 & 0 & 0 & 0 \\
\hline \multirow{2}{*}{ Asthma } & 20 & 15 & 14 & 13 & 11 & 10 & 14 & 16 & 8 & 19 & 9 & 11 & 10 & 21 & 15 & 14 \\
\hline & 0 & 0 & 0 & 0 & 0 & 0 & 0 & 0 & 0 & 0 & 0 & 0 & 0 & 0 & 0 & 0 \\
\hline \multirow{2}{*}{ Appendicitis } & 94 & 75 & 94 & 91 & 86 & 111 & 93 & 107 & 90 & 73 & 92 & 95 & 87 & 90 & 103 & 72 \\
\hline & 1 & 0 & 0 & 0 & 1 & 0 & 0 & 0 & 0 & 0 & 0 & 0 & 1 & 1 & 0 & 0 \\
\hline \multirow{2}{*}{ Abdominal hernia } & 105 & 67 & 74 & 83 & 73 & 58 & 73 & 49 & 107 & 66 & 89 & 82 & 76 & 67 & 94 & 74 \\
\hline & 1 & 0 & 0 & 1 & 1 & 0 & 2 & 3 & 1 & 0 & 0 & 0 & 0 & 2 & 1 & 2 \\
\hline \multirow{2}{*}{$\begin{array}{l}\text { Cholelithiasis and } \\
\text { cholecystitis }\end{array}$} & 82 & 77 & 92 & 82 & 104 & 74 & 88 & 74 & 117 & 84 & 137 & 97 & 140 & 115 & 152 & 117 \\
\hline & 0 & 0 & 1 & 0 & 0 & 0 & 0 & 1 & 0 & 1 & 0 & 0 & 0 & 0 & 0 & 0 \\
\hline \multirow{2}{*}{$\begin{array}{l}\text { Maternal and } \\
\text { perinatal mortality }\end{array}$} & 1015 & 1119 & 998 & 1019 & 905 & 1132 & 1045 & 1153 & 1108 & 1108 & 1054 & 1194 & 1080 & 1301 & 1195 & 1288 \\
\hline & 0 & 0 & 0 & 0 & 0 & 0 & 0 & 1 & 0 & 0 & 0 & 0 & 1 & 1 & 1 & 0 \\
\hline \multirow{2}{*}{$\begin{array}{l}\text { Osteomyelitis and } \\
\text { periostitis }\end{array}$} & 3 & 2 & 6 & 3 & 5 & 5 & 6 & 3 & 11 & 7 & 6 & 4 & 7 & 2 & 5 & 7 \\
\hline & 0 & 0 & 0 & 0 & 0 & 0 & 0 & 0 & 0 & 1 & 0 & 0 & 0 & 0 & 0 & 0 \\
\hline Total & 1744 & 1720 & 1617 & 1533 & 1507 & 1690 & 1616 & 1670 & 1745 & 1619 & 1671 & 1704 & 1697 & 1837 & 1850 & 1835 \\
\hline IOtal & 13 & 6 & 6 & 8 & 6 & 6 & 9 & 12 & 10 & 10 & 10 & 11 & 11 & 15 & 8 & 7 \\
\hline
\end{tabular}

Note: White boxes correspond to the number of admissions, while the shaded boxes represent the number of deaths for each pathology.

Comparison of total deaths in semesters 14 and 16 by chi-square test: $2.085, P$ value $=.000$.

Abbreviation: HI, health indicators.

was applied to calculate tendency. This result verified that the behavior of this tendency is strongly marked by these pathologies.

The RVR of the series fluctuated from -20 to +20 throughout the study period, except for the maximum value of semester 7 and the minimum values of semesters 2,15 , and 16 .

Figure 1 shows the arithmetic mean, tendency, and deseasonalized series for AM, and Figure 2 shows them only for hypertension. The curve of the tendency "softened" the extreme values of the mean and showed an ascending behavior with a first segment of slight oscillations, marked by increases. The second phase showed little variation until the end, when the values began to rise again. The last phase, the post-intervention period, was characterized by a pronounced decrease. The deseasonalized series and tendency have similar profiles; however, they differ in that the deseasonalized series includes tendency, the cycles, and residual variations, while tendency includes only the cycles. In this figure, the tendency shows a generally ascending behavior until semesters 15-16, when it changes radically. The results of the logistical regression, which indicate that in semester 16, from July to December 2015, the "expected" death rate would have been $0.70 \%$ if no intervention program had taken place. The percentage of mortality due to $\mathrm{HI}$ during the study period shows a progressive increase in deaths which reaches $0.53 \%$ during semester 14 (corresponding to the period July to December 2014), the period just prior to the training course. Clearly, without the intervention, the percentage of deaths would have potentially increased a further $0.17 \%$.

\section{Discussion}

Time-series analysis is one of the most common methods used in epidemiologic vigilance. It reduces uncertainty in decision-making and quickly detects new needs or the persistence of old problems. ${ }^{13}$ In studying mortality caused by $\mathrm{HI}$ pathologies, it is used to model those mechanisms which have generated the observed series and "predict" future values. ${ }^{14}$ 
Table 3. Values Calculated in the Time-Series Analysis of Mortality Due to HI Pathologies, and Observed Trend in The Pathology Hypertension and Cerebrovascular Diseases Over the 15- and 16-Semester Studied Period, 2008-2015

\begin{tabular}{|c|c|c|c|c|c|c|c|}
\hline \multirow[b]{2}{*}{ Year } & \multicolumn{6}{|c|}{ Time-Series on Mortality Due to HI Pathologies } & \multirow{2}{*}{$\begin{array}{c}\text { Trend of Hypertension } \\
\text { and Cerebrovascular } \\
\text { Diseases }\end{array}$} \\
\hline & Semester & $\begin{array}{c}\text { Arithmetic Mean of } \\
\text { Original Series } \\
\end{array}$ & Trend & $\begin{array}{c}\text { Rolling } \\
\text { Average Rate }\end{array}$ & $\begin{array}{c}\text { Deseasonalized } \\
\text { Series } \\
\end{array}$ & $\begin{array}{c}\text { Relative } \\
\text { Variation Rate } \\
\end{array}$ & \\
\hline \multirow{2}{*}{2008} & 1 & 1.08 & & & 1.15 & & \\
\hline & 2 & 0.50 & & & 0.47 & -59.13 & \\
\hline \multirow{2}{*}{2009} & 3 & 0.50 & 0.65 & 77.52 & 0.53 & 12.77 & 6.00 \\
\hline & 4 & 0.67 & 0.54 & 123.50 & 0.63 & 18.87 & 5.25 \\
\hline \multirow{2}{*}{2010} & 5 & 0.50 & 0.59 & 85.47 & 0.53 & -15.87 & 5.50 \\
\hline & 6 & 0.50 & 0.54 & 92.17 & 0.47 & -11.32 & 4.75 \\
\hline \multirow{2}{*}{2011} & 7 & 0.75 & 0.56 & 133.33 & 0.80 & 70.21 & 5.50 \\
\hline & 8 & 1.00 & 0.75 & 133.33 & 0.93 & 16.25 & 6.25 \\
\hline \multirow{2}{*}{2012} & 9 & 0.83 & 0.90 & 92.74 & 0.88 & -5.38 & 6.25 \\
\hline & 10 & 0.83 & 0.87 & 95.13 & 0.77 & -12.50 & 7.25 \\
\hline \multirow{2}{*}{2013} & 11 & 0.83 & 0.83 & 100.00 & 0.88 & 14.29 & 8.00 \\
\hline & 12 & 0.92 & 0.85 & 107.92 & 0.86 & -2.27 & 8.25 \\
\hline \multirow{2}{*}{2014} & 13 & 0.92 & 0.90 & 102.51 & 0.98 & 13.95 & 8.75 \\
\hline & 14 & 1.25 & 1.00 & 124.69 & 1.17 & 19.39 & 9.00 \\
\hline \multirow{2}{*}{2015} & 15 & 0.67 & 1.02 & 65.53 & 0.71 & -39.32 & 8.00 \\
\hline & 16 & 0.58 & 0.79 & 73.19 & 0.54 & -23.94 & 5.75 \\
\hline
\end{tabular}

Abbreviation: $\mathrm{HI}$, health indicators.

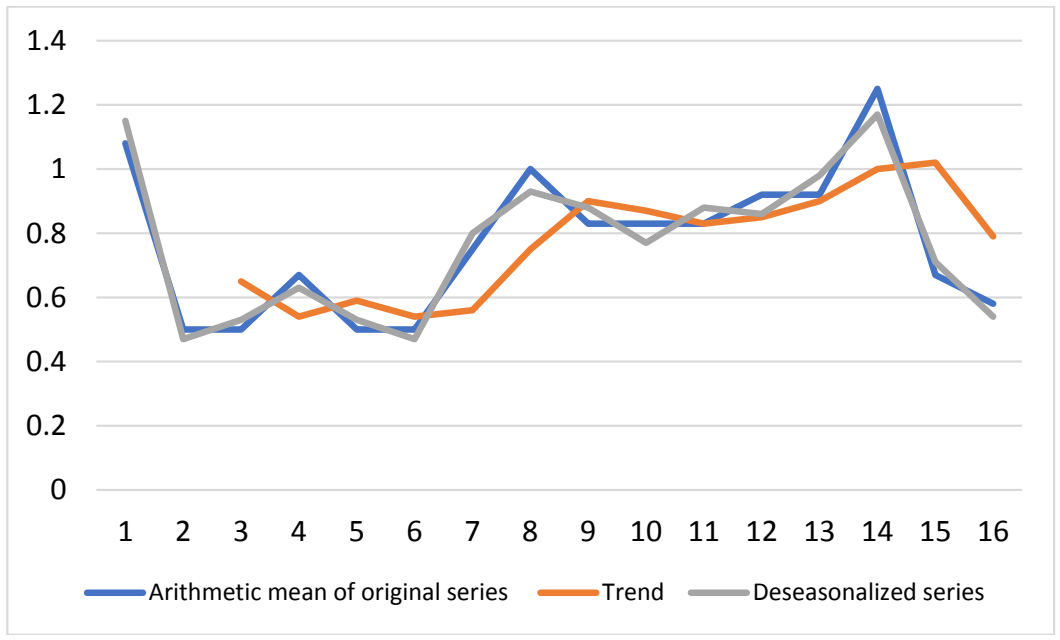

Figure 1. Original Series, Deseasonalized Series, and Trend of the Time Series on HI Mortality; 2008-2015 by Semesters.

This analysis, however, tacitly accepts that the factors which generated the deaths will continue to behave in the same manner, but in fact they go through variations. The health sector is dynamic; it controls its deficiencies and changes its action protocols. ${ }^{13}$ Despite this reflection, timeseries studies assume a certain stability in the determining factors, so short-term predictions make sense. Accessibility to these estimated values would facilitate decision-making and help define lines of action to address healthcare challenges. ${ }^{13,14}$

In the analysis of tendency, the results showed an ascending behavior during the 2008-2014 period, a characteristic which is conserved in the deseasonalized series. The general rate of seasonal variation was 6 points below the annual average in the semesters from January to June throughout the study period. In the semesters from July to December, the rate was 7 points above the annual average. In general, the variation rates fluctuated in a range from -20 to +20 from one semester to the next, with a few exceptions observed over the course of the study.

The series forms part of a continuous process and was studied for a relatively short period of time. However, the changes observed in the behavior of the series after applying the intervention program suggested a positive effect, with reductions in mortalities caused by $\mathrm{HI}$ pathologies. First of all, the binary logistical regression showed a $0.70 \%$ of "expected" deaths for semester 16 (corresponding to the period between July and December 2015, the phase following the training session). In semesters 1-14 (January 1, 2008 to December 31, 2014), the phase preceding the training session, the AM rate reached $0.53 \%$. Furthermore, a statistically significant difference was observed in the number of deaths between semester 14 and semester 16 $(P=.000)$. 


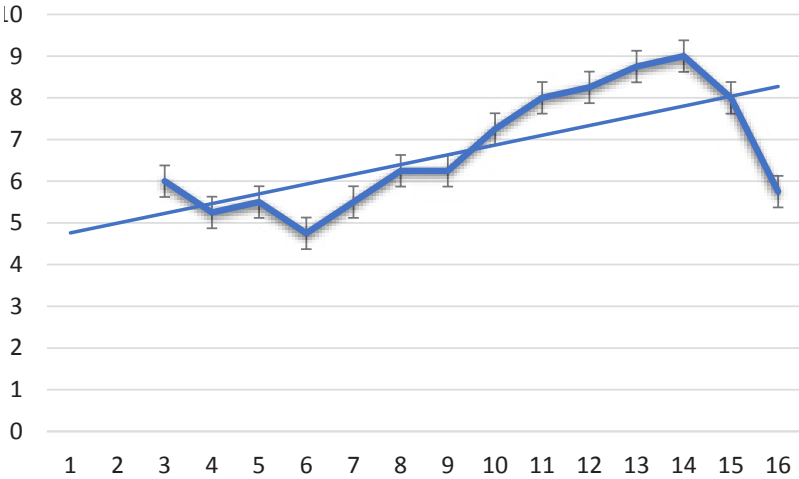

Figure 2. Trend of the Time Series on Mortality Due to Hypertension and Cerebrovascular Diseases; 2008-2015.

The importance of the intervention program and its effect in reducing mortalities from HI causes is supported by the following arguments. First, the priority topics to be dealt with in training seminars were defined by healthcare professionals who provide services which are directly or indirectly related to HI pathologies. The staff's participation was dynamic, as they showed keen interest in searching for strategies to optimize their professional performance.

Secondly, the incidence of admissions due to $\mathrm{HI}$ pathologies in the last three semesters of the study period remained relatively stable; thus, the decrease in the number of deaths was not significantly affected by the number of admissions.

Finally, 8 or 9 deaths from hypertension and cerebrovascular diseases were observed each semester from the first semester of 2011, a number which changed abruptly to 5 in the first semester of 2015 (when the intervention took place). During the last semester studied, after the intervention took place, 4 deaths occurred. All deaths corresponded to the same age group throughout the study period, and there were no changes among the number of staff nor in the hospital service infrastructures that attended these pathologies.

A similar study in the British Health Foundation created a pioneering program to be developed in two phases. The first was carried out in 2004 in 4 hospitals, and the second included 20 hospitals and took place in 2006. In both centers, health professionals reviewed issues related to the prevention of complications in hospital patients. At the end of 2006, the first group of hospitals had eliminated nosocomial pneumonia, and medication expenses decreased by $78000 .^{15}$

Finally, the authors reiterate a fundamental element defined by Aiber and Aranaz, which is "health assistance" risk. ${ }^{16,17}$ According to these authors, maximum patient safety requires sufficient knowledge in order to eliminate unnecessary risks and prevent those that may be inevitable. In this regard, warning systems and epidemiological studies that allow changes at different levels of the health system are important. ${ }^{18}$

The current approach to adverse effects in clinical practice is to analyze accidents as part of a system.

\section{Research Highlights}

\section{What Is Already Known?}

It is known that avoidable hospital mortality is a set of pathologies whose hospital mortality should be avoided according to scientific research alone.

\section{What This Study Adds?}

This study confirms that the practical programs of training personnel in the treatment of AM pathologies reduce mortality.

Researchers at the university hospitals of Salamanca and Alicante consider that even the simplest negative effects are not due to a sole mistake. Rather, they are generally a combination of multiple errors that may be related to deficiencies of the system or be directly dependent on the professional. ${ }^{19}$

The present study developed a strategy which is garnering excellent results in Holland and the United States, ${ }^{20-22}$ motivating and providing incentives to healthcare personnel to improve their professional activity and decrease mortality due to HI pathologies.

The methodological design was limited to the hospital stay. In addition, the follow-up period after the intervention should have been longer; an effect may have been observed if the post-study monitoring had lasted longer.

\section{Conclusion}

This training program of the hospital staff made it possible to reduce AM by half, which makes it possible to affirm that these programs improve the quality of care and should be applied.

\section{Authors' Contributions}

All authors contributed equally to this research.

\section{Conflict of Interest Disclosures}

No conflicts of interest are declared.

\section{Ethical Approval}

This study was approved by the Ethics Committee of the participating hospital.

\section{References}

1. Rutstein DD, Berenberg W, Chalmers TC, Child CG, Fishman AP, Perrin EB. Measuring the quality of medical care. A clinical method. N Engl J Med. 1976;294:582-588.

2. Charlton JR, Hartley RM, Silver R, Holland WW. Geographical variation in mortality from conditions amenable to medical intervention in England and Wales. Lancet. 1983;1:691-696.

3. Charlton JR, Velez R. Some international comparisons of mortality amenable to medical intervention. Br Med J (Clin Res Ed). 1986;292(6516):295-301.

4. Holland W. European Community Atlas of Avoidable Death. Oxford: Oxford University Press; 1988.

5. Holland W. Avoidable death as a measure of quality. Qual Assur Health Care. 1991;2:227-233.

6. Benavides FG, Orts R, Perez S. Adding years to life: effect of avoidable mortality on life expectancy at birth. J Epidemiol Community Health. 1992;46:394-395. 
7. Gispert R, Torne MD, Arán BM. The efficiency of the Health System in Spain. Gac Sanit. 2006;20 Suppl 1:117-126.

8. Melchor I, Nolasco A, Garcia-Senchermes C, et al. Avoidable mortality. Changes in the new century? Gac Sanit. 2008;22:200209. doi:10.1157/13123965.

9. EC Working Group on Health Services and "Avoidable Deaths". European Community Atlas of "Avoidable Death". 1991.

10. Moauro F, Savio G. Temporal disaggregation using multivariate structural time series models. Econom J. 2005;8(2):214-234.

11. Beck N, Katz JN. What to do (and not to do) with time-series cross-section data. Am Polit Sci Rev. 1995;89(3):634-647.

12. Crighton EJ, Muhammad MM, Ross EG. A population based time series analysis of asthma hospitalisations in Ontario, Canada: 1988 to 2000. BMC Health Serv Res. 2001;1:7. doi:10.1186/1472-6963-1-7.

13. Gisele CM. Pronósticos de mortalidad por enfermedades no transmisibles seleccionadas. Rev Cubana Hig Epidemiol. 2008;46(3).

14. Martínez NF. Vigilancia Epidemiológica. 1st ed. Madrid: McGraw-Hill Interamericana; 2004:104

15. Aibar C, Aranaz J. Can adverse effects related to hospital treatment be avoided? An Sist Sanit Navar. 2003;26(2):195209.

16. Aranaz JM, Aibar C, Galan A, et al. Health assistance as a risk factor: side effects related to clinical practice. Gac Sanit. 2006 20:41-7. doi: 10.1157/13086025.

17. Kuhn AM, Younberg BJ. The need for risk management to evolve to assure a culture of safety. Qual Saf Health Care. 2002;11:158-62. doi:10.1136/qhc.11.2.158.

18. Otero LM. Errores de medicación y gestión de riesgos. Rev Esp Salud Publica. 2003;77:527-40.

19. Institute of Medicine. To Err Is Human: Building a Safer Health System. Washington DC: National Academy Press; 1999. http://www.csen.com/err.pdf.

20. American Society for Healthcare Risk Management. Strategies and tips for maximizing failure mode and effect analysis in an organization. J Healthc Risk Manag. 2002;22:9-12. doi: 10.1002/jhrm.5600220304.

21. McDonough JE. Proactive hazard analysis and health care policy. New York: Milbank Memorial Fund; 2002. http://www. milbank.org/reports/HazardAnalysis.pdf.

22. Habraken MP, Van der Schaaf TW, Leistikow IP, ReijndersThijssen MJ. Prospective risk analysis of health care processes: a systematic evaluation of the use of HFMEA in Dutch health care. Ergonomics. 2009;52:809-819. doi: $10.1080 / 00140130802578563$

\section{Appendix. Correction Factors}

The correction factors were calculated for use in a log-linear model of general form:

$$
\log \left(E\left(O_{t}\right)=\log \left(p_{t}\right)+c_{t}+f\left(X_{t}\right)\right.
$$

in which ' $t$ ' is the year between $T_{1}$ and $T_{2}$; $c_{t}$ is the correction factor; and $f\left(X_{t}\right)$ is a function of independent variables. The correction factors were set so that the last values of the corrected mortality rates were equal to the exact mortality rates, i.e. $C_{T 2}=0$. This choice was based on the supposed superior quality and between-country comparability of the most recent year's data.

The foregoing results in the following definition of the correction factors $c_{t}$ :

$$
\text { For } t \in\left[T_{1}, T_{2}\right], c_{t}=\sum_{t^{\prime} \in S, t^{\prime}<t} d_{t^{\prime}}-\sum_{t^{\prime} \in S} d_{t^{\prime}}=-\sum_{t^{\prime} \in S, t^{\prime} \geq t} d_{t^{\prime}} .
$$

The estimate of $c_{t}$ was then directly obtained from the estimates of $S$ and $d_{t}$ detailed earlier.

A corrected version of the log mortality rate was then obtained as: $L_{t}^{c o r}=L_{t}-c_{t}$ 